Revue d'histoire de l'Amérique française

REVUE D'HISTOIRE DE L'AMÉRIQUE FRANÇAISE

\title{
Mesure de l'influence du chanoine Lionel Groulx sur son milieu
}

\section{François-Albert Angers}

Volume 32, numéro 3, décembre 1978

Lionel Groulx, $100^{\mathrm{e}}$ anniversaire de sa naissance, 1878-1978

URI : https://id.erudit.org/iderudit/303715ar

DOI : https://doi.org/10.7202/303715ar

Aller au sommaire du numéro

Éditeur(s)

Institut d'histoire de l'Amérique française

ISSN

0035-2357 (imprimé)

1492-1383 (numérique)

Découvrir la revue

Citer cet article

Angers, F.-A. (1978). Mesure de l'influence du chanoine Lionel Groulx sur son milieu. Revue d'histoire de l'Amérique française, 32(3), 357-384.

https://doi.org/10.7202/303715ar d'utilisation que vous pouvez consulter en ligne.

https://apropos.erudit.org/fr/usagers/politique-dutilisation/ 


\section{MESURE DE LINFLUENCE DU CHANOINE LIONEL GROULX SUR SON MILIEU*}

FrANÇOIS-ALbERT ANGERS

HEC

Montréal

L'appréciation qui suivra du rôle du chanoine Lionel Groulx et de son influence sur son milieu sera tellement favorable qu'il est sans doute important, pour que le lecteur puisse en juger la valeur, de situer l'auteur par rapport au personnage. L'abbé Groulx a eu des admirateurs et des disciples inconditionnels. Plusieurs furent des jeunes, dont il n'était l'aîné que d'une demi-génération au début, et qui sont toujours restés fidèles dans leur admiration d'ailleurs grandissante. D'autres jeunes se sont joints à cette première cohorte, alors que lui, il les distançait d'âge de génération en génération sans que se creusât entre lui et eux le moindre fossé, au moins jusque vers les années 50 . On peut suspecter ceux-là d'être difficilement objectifs dans leurs jugements sur lui; d'avoir une tendance naturelle à le voir grand à travers leur affection et leur enthousiasme de toujours, plus qu'en fonction de la réalité des faits. Ce n'est pas tout à fait mon cas.

J'ai connu l'abbé Groulx pour la première fois un soir de septembre 1938, alors que $\mathrm{j}$ 'assistais à ma première réunion comme «directeur» de la Ligue d'Action nationale. J'y étais entré d'une façon d'ailleurs plutôt insolite. Non pas sans conviction, mais dans l'immédiat en raison d'un conflit qu'avait suscité à l'intérieur du groupe un de mes articles ${ }^{1}$. Esdras Minville et André Laurendeau souhaitaient fort m'associer à la Ligue, mais j'étais réticent, en tant

* Ce texte a fait l'objet d'une communication à un colloque tenu au Collège de Valleyfield, les 2, 3, 4 mai 1978. Ont été ajoutés à la présentation originelle, l'introduction sur mon expérience personnelle avec l'abbé Groulx et un développement plus poussé de la discussion sur nationalisme et catholicisme chez Groulx. Ces sujets avaient fait l'objet de questions de la part des participants au colloque.

1 L'article intitulé «L'économique", publié en octobre 1937, sous le titre Pour une politique nationale.

RHAF, vol. 32, no 3, décembre 1978 
que jeune économiste, de m'identifier aussi étroitement avec ce que d'autres me conseillaient d'éviter : les chapelles. J'étais consentant à fournir mon apport comme collaborateur régulier, mais extérieur et sur les questions économiques, à une oeuvre dont je partageais les idées en général, mais sans vouloir en porter l'étiquette. À l'occasion de l'incident mentionné, Laurendeau me fit valoir, avec succès, que quant à collaborer régulièrement, ce serait prendre une assurance sur ma liberté d'expression que d'être à l'intérieur et de défendre moi-même mes positions aux réunions de la Ligue, plutôt que d'avoir à subir toutes les tractations qu'avait provoquées mon premier article à la revue. Songeant avant tout, à ce moment-là, à ma carrière d'économiste et à ses exigences, je n'étais donc pas dans l'état d'ardeur du croisé patriote, qui était typique des jeunes de l'entourage idéologique de l'abbé Groulx.

N'étant pas de la région de Montréal, mais de celle de Charlevoix, je n'avais pas participé à toute l'activité nationaliste qui accompagnait la montée de l'abbé Groulx depuis 1917, et j'en connaissais alors peu de chose. J'étais actif dans l'A.C.J.C. de ma région, mais il était peu question alors de l'abbé Groulx dans ces lieux éloignés du Québec. C'était Henri Bourassa qui était le grand prophète. De 1928 à 1937, j'avais cependant gagné Montréal, où j'étudiaì d'abord à l'École des H.E.C., puis je restai comme stagiaire à la même École. Je m'étais inscrit à un cercle montréalais d'A.C.J.C., le cercle Louis Colin, à la requête de mon professeur de philosophie, l'abbé Lucien Pineault ; mais j'y participais peu, m'occupant presque exclusivement, et d'une façon intensive, de mes études. Je ne fus donc pas engagé dans ce mouvement montréalais des années 30 qui conduisit, sous l'égide de l'abbé Groulx, à la fondation des Jeune-Canada ; et je ne connaissais que deux de ceuxlà - Gérard Filion et Thuribe Belzile - parce qu'ils étaient étudiants aussi à l'École des H.E.C., sans rien savoir ou connaître d'ailleurs de leurs activités nationalistes avant la fondation des Jeune-Canada. Lors de cette fondation, à l'automne 1932 je crois, j'avais en fait quitté Montréal pour un stage de cure au Sanatorium du Lac Edouard. J'en appris là, la nouvelle, par les journaux, de sorte que je n'avais pu être sollicité d'y appartenir, si tant est qu'on avait pensé à me voir à ce sujet, ce qui est plus qu'improbable.

En 1938, quand je rencontrai l'abbé Groulx pour la première 
fois, la seule oeuvre de lui que j'avais lue était son roman $A u C a p$ Blomidon. J'en savais cependant plus que cela sur lui en tant que lecteur, pas toujours enthousiaste à cette époque d'ailleurs, du Devoir. Mais cela ne m'avait pas particulièrement arrêté, l'estimant "séparatiste", ce qui ne correspondait pas du tout à mes opinions du temps. Entre Laurendeau et moi, qui avons été très amis pendant de nombreuses années, et travaillé ensemble d'une façon quotidienne jusqu'après son entrée au Devoir, il y eut ceci de curieux que nous avons évolué exactement en sens contraire dans nos perceptions et nos convictions nationalistes. Et ce qui l'arrêtait le plus fut ce qui me convainquit le plus: l'économique.

Je devais cependant me trouver rapproché de Groulx par cette amitié avec Laurendeau, qui prit naissance lorsque nous nous connûmes à Paris, où nous étudiions tous les deux, de 1935 à 1937. André Laurendeau était alors un des disciples fervents et le disciple préféré de l'abbé Groulx. Nos conversations tournaient donc fréquemment vers lui. Entre autres, André avait à coeur de me démontrer que l'abbé Groulx n'était pas séparatiste; j'argumentais très fort, d'après mes perceptions de lecteur du Devoir, pour prouver et marquer ma méfiance à son égard. C'est donc à travers trente ans de travail en commun avec l'abbé Groulx, à L'Action nationale, que va se former, et graduellement, mon opinion et mes sentiments de haute admiration pour un homme dont je pris conscience qu'il était authentiquement grand à tous égards.

Après mon entrée à L'Action nationale, je suis donc resté plutôt très réservé à son égard pendant plusieurs années. Il n'était plus dans la lutte active à ce moment-là, s'étant " retiré " dix ans plus tôt (en 1927) de la ligne de feu pour se consacrer tout d'abord à son ouvre d'historien. Il fallut du temps avant que j'en vienne à une connaissance suffisante de son oeuvre pour apprécier correctement les opinions et les attitudes alors épisodiques qu'il nous manifestait, selon les événements, aux réunions mensuelles de la Ligue. La partie politique de son oeuvre, notamment son travail à L'Action française, son ampleur et sa richesse, je n'en prendrai pleine conscience qu'en 1962, alors que comme directeur de la revue L'Action nationale, à mon tour, je préparai le numéro spécial sur « 50 ans de nationalisme positif $»^{2}$. Je dus alors lire, en particulier, l'ensemble de

2 L'Action nationale (mars-avril 1963). 
cette partie de son ouvre. Entre-temps, après 1938, j'avais naturellement suivi de près l'expression plus finie de sa pensée, quoique toujours épisodique et partielle, qu'il révélait dans ses articles et conférences, publiés ensuite en volumes ou en brochures. C'est donc dans la perspective d'un esprit longtemps critique, mais progressivement conquis par l'évidence de la grandeur d'âme, de coeur et d'esprit, que se sont formés les jugements que je porte aujourd'hui sur la carrière et l'ouvre du chanoine Groulx.

Or, mesurer l'influence qu'il a exercée sur son milieu, c'est d'abord poser le problème général de la place que peut prendre une pensée créatrice dans un milieu politique et social. Car «son action", il l'a lui-même lucidement voulue "intellectuelle "3. Il aura tenté de l'exercer dans deux champs: celui de l'histoire, en tant qu'historien précisément; celui de la politique au sens très large du terme, par la propagation d'idées très étroitement reliées au destin particulier d'un peuple, son "petit peuple", le peuple canadienfrançais.

Encore, faut-il d'abord démontrer l'existence d'une pensée originale pour justifier une influence de pensée créatrice. Puis en établir la portée sur les manières de penser et les actes des contemporains et des générations successives. Une influence donc, dont l'image serait le déploiement de l'onde circulaire, tendant à se propager indéfiniment, à partir d'un point d'impact - une pierre jetée à l'eau - qui vient rompre la tranquillité d'un milieu ambiant. Exemple qui fait sentir la difficulté de certaines démonstrations, en raison même du fait que, dans le jeu des phénomènes sociaux, on ne se trouve jamais dans la position de l'agent qui a lancé la pierre et qui observe de haut et dans une perspective d'ensemble, l'effet produit dans le miroir du lac. Nous sommes, au contraire, à peu près toujours dans la position d'une des gouttes d'eau, quelque part dans le mouvement de propagation de l'onde. Les causes et les relations qui nous frappent sont davantage celles qui nous sont les plus proches. 
Ainsi, au plan de l'action politique, on accordera plus volontiers la palme de l'influence à ceux qui ont réalisé les choses plutôt qu'à ceux qui les ont pensées. À ceux «qui se sont mouillés ", comme on aime à dire, c'est-à-dire qui se sont fait élire, qui sont surtout devenus ministres, et ont posé des gestes ayant des effets concrets immédiats. Or, en cela, Groulx apparaît plutôt comme l'homme qui a été contre ce qui s'est fait en son temps, et qu'il n'a pas pu changer en ce temps. Cela le situe, pour les observateurs superficiels, dans un constat d'échec perpétuel. Il faut donc suivre attentivement le cheminement de ses idées dans le temps pour en mesurer la portée. C'est tout au plus une poignée d'hommes qu'il entraîne tout d'abord autour de lui, et qui oeuvrent dans un climat hostile au système de pensée qu'il développe.

Pendant toute la vie de l'abbé Groulx, ces hommes ne prendront jamais le pouvoir qui leur aurait permis de réaliser ses idées. Leur influence se manifeste par leur portée électorale à long terme, qui obligera les politiciens adverses à les intégrer petit à petit dans leurs programmes, et presque toujours à leur corps défendant. $\mathrm{Ce}$ n'est que tout récemment, et après plusieurs essais infructueux, l'échec des deux tiers partis successifs de l'Action libérale nationale (1936) et du Bloc Populaire (1948) - que naîtra un parti incarnant ouvertement une pensée nationaliste authentique : le Parti québécois ; et qui la portera au pouvoir. En cours de route, le nombre des disciples, des admirateurs ou des adhérents à ces idées croît ; et c'est alors à ceux-là qu'au fur et à mesure du temps, nous sommes portés à attribuer tout le mérite des fruits, même de pensée, oubliant l'événement de plus en plus lointain du jet initial de la pierre. Quoique dans le cas de Lionel Groulx, le jet de la pierre, son travail intellectuel, s'accompagnera d'une inlassable activité de propagation de ses idées par voie d'articles, de conférences, d'entrevues, etc.

Dans le domaine de l'histoire du Canada, son influence est plus facile à mesurer, car tout se passe ici en milieu intellectuel, dans le monde des historiens. Ce qui est en cause, c'est une conception de l'histoire. L'originalité de l'apport se mesure alors objectivement par des analyses de textes, des comparaisons d'auteurs, avant et 
depuis Groulx. Sur ce point, personne aujourd'hui, semble-t-il, ne conteste le rôle majeur du chanoine Groulx. Quelle fut la nature de ce rôle? Il m'apparaît se situer plus sur le plan de l'interprétation de notre histoire, selon ce qu'on peut estimer être "sa vérité ", que sur le plan de la méthodologie propre de l'histoire. Et cela s'entend non seulement quant à la vérité pour Groulx, au sens pirandellien de "chacun sa vérité», mais à travers sa perception, d'une vérité objective intrinsèque aux événements qui tissent notre histoire. Il est courant aujourd'hui, chez les historiens, de constater qu'avant Groulx, l'histoire des Canadiens français s'écrivait comme celle d'un tronçon de peuple, perdu dans un continent à la suite d'une conquête, et dont on exaltait le passé mais considérait le sort comme scellé dans la défaite. La préservation de la langue et de la culture était envisagée comme un fait de "survivance ", mais dans la perspective d'un destin fermé. D'où une tendance des historiens, de nos historiens mêmes, à célébrer les bienfaits de la Conquête dans l'acceptation et pour la valorisation du statu quo. Alors que d'autres, même sympathiques aux perceptions historiques de Groulx, nous ont estimé "condamnés à survivre", donc à vivoter, sans autre espoir.

Groulx brise la circularité de ces interprétations, tendant vers le culte de la résignation face à l'avenir, pour y substituer le sens de l'«essor souverain" d'un destin de peuple ayant ses lettres de créance comme tel dans la ligne de sa propre histoire, et cela en toute objectivité historique 4 . C'était révolutionner la conception de notre histoire. Et les fruits de cette révolution me paraissent indéniables chez toutes les générations d'historiens qui vont suivre. Mais je ne veux pas m'aventurer davantage dans ce domaine de spécialistes. Laissons-en à ceux-là l'exploration et la discussion.

C'est donc à l'influence politique de Lionel Groulx que sera consacrée la suite du présent texte. Il y a eu là une "carrière politique " véritable, parallèle et équivalente au moins en importance à la carrière plus connue et plus exaltée d'historien. Il l'a exercée,

4 L'Action française (janvier 1921). Voir aussi Lionel Groulx, Pourquoi nous sommes divisés (1943), 6-7 (cité dans G.-E. Giguère, Lionel Groulx-biographie (éd. Bellarmin, 1978), 82. 
répétons-le, sur le plan d'une "action intellectuelle", donc de la conception et de la propagation d'une pensée génératrice d'action politique. Le point important ici à ce sujet, nous l'avons dit précédemment, c'est de cerner d'abord l'apport "créateur" de cette pensée.

Dans un texte que publiait L'Action nationale de mai 1978, un texte de célébration du centenaire de naissance de Groulx, je donnais à cette action une portée qui justifierait de considérer cet homme comme notre libérateur, à la façon d'un Gandhi pour l'Inde. Dans un colloque de ton plus scientifique, c'est le mode qu'il faut dégager autant que la conséquence finale. Il faut penser aussi à ce que l'intéressé a voulu réaliser, autant qu'à ce que nous estimons avoir été le résultat ultime de ses réalisations. En ce sens, c'est sans doute aller plus en profondeur de dire qu'il a voulu être l'éducateur d'un peuple pour le préparer à une libération dont il estimait luimême qu'elle n'était pas mûre en son temps ${ }^{5}$. En fait, il a plus qu'éduqué; il a pratiquement façonné un peuple qui est maintenant en marche vers sa libération. Ce sont les idées qu'il a élaborées, développées, propagées, qui ont amené le peuple canadien-français tel qu'il était au début du XXe siècle, à se considérer comme une nation ayant droit à la libre disposition d'elle-même.

Bien sûr, ce ne fut pas une création ex nihilo. Il aurait été le premier à l'affirmer hautement de son vivant contre toute exagération de son rôle par un disciple trop fervent. Avant Groulx, il y avait eu toute notre histoire où il puise justement la conviction de ce que nous sommes. Son objectif n'est pas de faire passer une idée de l'imaginaire au réel, mais de nous révéler notre vraie réalité ensevelie sous les cendres de la Conquête, de la répression de 1837 et de la propagande politique d'un demi-siècle de Confédération.

Certes, au temps de sa jeunesse et de sa période de formation, il y avait des esprits qui perpétuaient cette sensibilité à l'être national, tel Jules-Paul Tardivel. Mais ceux-là mêmes constituaient, selon ma perception de l'état des choses à ce moment-là, plutôt des résidus rares d'une conception profondément décadente en termes d'importance actuelle en étendue. Ils étaient le feu quasi éteint sous des cendres devenues passablement froides. Selon cette perception de l'état de conscience du peuple canadien-français dans son ensemble

5 cf L'Action nationale (mai 1978) : 709. 
au début de $\mathrm{XXe}$ siècle, la place unique, et tout d'abord quasi exclusive, que Groulx va tenir dans la restauration de l'idée nationale intégrale apparaît aujourd'hui d'une portée incommensurable. Là, en effet, origine la réanimation de la conscience actuelle que le Québec a de son destin de nation. C'est bien l'onde circulaire provoquée par le jet d'une pierre dans la surface tranquille du lac, avec la différence des amplifications ultérieures engendrées par les effets psychologiques que déclenche le mouvement même.

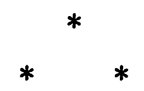

L'appréhension du phénomène suppose donc une prise de conscience aiguë et réaliste de la situation où se trouvait le Canada français lorsque Lionel Groulx intervient. La nation canadiennefrançaise existe, aperçoit-il. Elle a été reconnue, très officiellement, dès le milieu du XIXe siècle, par cet observateur étranger dont tout le monde se plaît à reconnaître l'intelligence et la lucidité, même dans le désaccord sur ses conclusions: lord Durham. L'on sait qu'arrivé à Québec pour enquêter sur les événements de 1837, il écrira : "Je m'attendais à trouver un conflit entre un gouvernement et son peuple: je trouvai deux nations en guerre au sein d'un même État; je trouvai une lutte non de principes mais de races. " Et il n'avait pas été seul à percevoir notre caractère de peuple bien identifié : à peu près tous les observateurs étrangers ont constaté la même chose.

Il existe à ce sujet, chez certains de nos historiens, une thèse qui veut la rébellion de 1837 n'avoir été qu'une affaire de "principes " de gouvernement, en pleine contradiction alors avec les constatations de lord Durham. Il y a là une bonne part de vérité documentaire, mais une large part d'erreur de perspective. De toute façon, cela importe moins pour ce qui nous concerne ici, car si nous étions vraiment alors si peu "nationalistes", le rôle de l'abbé Groulx se trouverait encore davantage magnifié, il aurait pratiquement alors inventé de toute pièce une nation qui vibre comme telle et vit aujourd'hui.

Il reste - et c'est ce que j'appelle l'erreur de perspective - que le nationalisme des patriotes de 1837 n'avait pas à s'exprimer de la même façon que notre nationalisme d'aujourd'hui, parce que le 
Québec du temps, en tant que Québec, était intact, avec son Assemblée législative propre et unique, quoique assujettie à un exécutif colonial nommé par Londres. Conquérir les libertés politiques de la suprématie de l'Assemblée législative et du principe du gouvernement responsable, c'était assujettir l'exécutif à cette assemblée contrôlée par le peuple canadien-français; et par suite, créer déjà un État français du Québec n'ayant plus de lien avec Londres que par le gouverneur. Le caractère nationaliste de la situation était aussi marqué par l'alignement des forces politiques à l'Assemblée, avec ses deux partis, dont l'un était le parti canadien-français et l'autre, le parti des marchands ou des Anglais.

Cette façon de voir l'avenir du Québec, on la retrouvera au moment de la Confédération, dans le conflit entre Dorion et Cartier, entre ceux qui préféraient un Québec colonie britannique indépendante des autres colonies, et ceux qui se faisaient les apôtres d'une Confédération où seraient respectés les "droits des minorités" et les "droits des provinces ». C'est au fond le même genre de clivage fondamental que l'on retrouvera vers 1920, entre l'abbé Groulx et Henri Bourassa. Non pas que l'abbé Groulx ait proposé un tel réarrangement des situations dans l'Empire, mais que la formule Dorion ait toujours eu sa sympathie et qu'il cherche autre chose dans la Confédération, contrairement à Bourassa, que la situation de minorité ethnique protégée dans ses droits par des garanties constitutionnelles de minorité privilégiée.

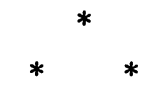

Donc nous étions une nation, et une nation bien consciente d'elle-même jusqu'à 1840 ; une nation en voie de se libérer progressivement du joug colonial par la conquête du gouvernement responsable dans un territoire où un tel pouvoir, répétons-le, aurait signifié le gouvernement par soi-même, donc un Québec français appelé à devenir aussi indépendant que l'est le Canada d'aujourd'hui. Mais au début du XXe siècle, à travers le jeu des forces politiques dans l'Union et dans la Confédération, ce sens d'une dignité nationale du citoyen, identifiée à l'affirmation d'une souveraineté française, s'est bien dilué, s'il n'est pas même à peu près disparu de la conscience populaire. Le fait français, au Québec même, ne s'exprime plus 
guère que comme un problème de "survivance" d'un "groupe ethnique " perçu comme minoritaire par rapport à l'ensemble du Canada. Quant au reste, la grande perception populaire de notre situation - et elle restera jusqu'à l'après-guerre 1939-1945 c'était: "Que voulez-vous qu'on fasse, nous appartenons à l'Angleterre ". Le Québec en est venu à se considérer comme un élément secondaire, dominé mais sans rémission et n'ayant rien de mieux à faire que d'être une province comme les autres, dans un grand tout canadien. Au point que nos hommes politiques, en général, et Bourassa lui-même, trouvent peu intéressante la scène politique "provinciale". Le prestige de faire de la politique est, à cette époque, à Ottawa; et passer de Québec à Ottawa constitue une forme de promotion certaine.

Cependant, en fin du XIXe siècle, divers événements bien connus ont soumis à un traitement de choc décevant ce peuple quasi prêt à se considérer dorénavant comme un groupe ethnique sans prétentions nationales fortes ${ }^{6}$. Alors, on se sent menacé dans la "survivance" comme minorité ethnique même, en dépit des assurances de nos chefs politiques que cette survivance serait assurée par nos attitudes "bonne ententistes".

Henri Bourassa sera le fer de lance de la lutte qui en résulte face à la démission même des autres politiciens devant les graves "violations " du "pacte confédératif". Mais pour réussir à restaurer le respect du "droit des minorités" et du "droit des provinces", il estime que le Québec doit se rallier totalement et pour toujours à la formule confédérative. Il ne voit pas l'utilité, au contraire il ne voit que des dangers, au réveil d'un nationalisme intégral. Selon lui, ce qui lui permettra de gagner la partie, c'est la garantie qu'il pourra donner aux Anglo-Canadiens de la fidélité des Canadiens français au Canada, si eux respectent nos «droits minoritaires", à la fois en. temps que minorité française dans les provinces anglaises, et que province de Québec reconnue en gros comme française et assurée de certains pouvoirs exclusifs de gouvernement tels que définis en 1867.

6 Pour ceux qui n'ont pas suffisamment de souvenirs historiques, rappelons qu'il s'est agi de la pendaison de Louis Riel, de l'abolition du français comme langue officielle du Manitoba et du refus de reconnaitre des droits scolaires aux francophones du NouveauBrunswick, du Manitoba et des Territoires, et de l'envoi de soldats pour aider l'Angleterre dans la guerre des Boers, en Afrique du Sud, préfiguration des deux crises de la conscription pour service militaire dans "les guerres de l'Angleterre" en 1917 et en 1942. 
Pour mener sa lutte, il fonde la Ligue nationaliste, en termes de nationalisme canadien, dirigé contre l'impérialisme britannique. L'absence d'un sentiment vraiment canadien chez les anglophones lui paraît la racine du mal. Jules-Paul Tardivel n'est pas d'accord et l'attaque sur ce point même, dans La Vérité du ler avril 1904. Il écrit :

... le nationalisme de la Ligue n'est pas notre nationalisme à nous... Notre nationalisme à nous est le nationalisme canadien-français. Nous travaillons, depuis 23 ans, au développement du sentiment national canadien-français : ce que nous voulons voir fleurir, c'est le patriotisme canadien-français; les nôtres, pour nous, sont les Canadiens français; la Patrie, pour nous, nous ne disons pas que c'est précisément la province de Québec, mais c'est le Canada français; la nation que nous voulons voir se fonder, à l'heure marquée par la divine Providence, c'est la nation canadienne-française.

Et Bourassa donne une "réponse amicale " à La Vérité, le 3 avril, dans Le Nationaliste, réponse qu'il synthétise finalement en adoptant la forme même dans laquelle Tardivel a résumé sa critique :

Notre nationalisme à nous est le nationalisme canadien, fondé sur la dualité des races et sur les traditions particulières que cette dualité comporte. Nous travaillons au développement du patriotisme canadien, qui est à nos yeux la meilleure garantie de l'existence des deux races et du respect mutuel qu'elles se doivent. Les nôtres, pour nous comme pour M. Tardivel, sont les Canadiens français; mais les Anglo-Canadiens ne sont pas des étrangers, et nous regardons comme des alliés tous ceux d'entre eux qui nous respectent et qui veulent comme nous le maintien intégral de l'autonomie canadienne. La Patrie, pour nous, c'est le Canada tout entier, c'est-à-dire une fédération de races distinctes et de provinces autonomes. La nation que nous voulons voir se développer, c'est la nation canadienne, composée des Canadiens français, et des Canadiens anglais, c'est-à-dire de deux éléments séparés par la langue et la religion, et par les dispositions légales nécessaires à la conservation de leurs traditions respectives, mais unies dans un attachement de confraternité, dans un commun attachement à la patrie commune.

Ici une parenthèse s'impose. Quelle a été exactement l'ouvre 
de Tardivel? Quelles thèses a-t-il développées au cours de ses longues années de juurnalisme au service de la cause canadiennefrançaise? Que retrouve-t-on dans l'abbé Groulx qui n'aurait pas été déjà dit par Tardivel et de la même façon ? C'est un aspect majeur dans la question de savoir quel fut l'apport spécifique de l'abbé Groulx à notre pensée nationaliste contemporaine. Le soussigné n'ayant pas de lumières particulières sur cette question la laisse tout simplement ouverte. Mais il lui apparaît que Tardivel constituait, au moment où Groulx entre dans sa vie active, le chef de file de la dernière tête de pont de l'école nationaliste d'avant 1840 ; et une dernière tête de pont fortement marginalisée et sans grande influence sur le cours effectif des événements. Quand Groulx entrera dans la lice pour vrai, environ 15 ans plus tard, l'action vigoureuse même de Bourassa aura encore démobilisé bien davantage les Canadiens français de cette optique.

Tout est donc à reprendre. Il ne s'agit plus uniquement de propager une idéologie déjà installée. Il faut la reformuler. La justifier intellectuellement autant qu'émotivement, car à l'instinct toujours présent manque la conviction, contrebattue depuis troisquart de siècle par l'idée canadienne régnante. Il y a à élaborer un tout doctrinal d'un nationalisme authentique, basé sur l'idée de souveraineté, pour servir d'instrument conceptuel d'émancipation. Cela est devenu indispensable pour rendre dynamiques les fondements instinctifs. Lionel Groulx s'y emploie consciemment et systématiquement, en même temps qu'il en organise la propagation d'abord avec un nombre restreint de collaborateurs et disciples, par l'intermédiaire d'une revue. L'idée fera ensuite son chemin par l'élargissement constant du cercle des adeptes. C'est pourquoi son influence ne se mesure pas à la méconnaissance dont faisaient preuve à son endroit, dans un film récent, les étudiants d'un CEGEP qui porte son nom. Car elle a quelque chose d'éternel en cela même qu'elle se propage indépendamment de toute référence à sa personne, sans qu'on sache que tout ou presque part de lui.

À l'occasion justement de la conférence que je prononçais à Québec, le 21 janvier dernier ${ }^{7}$, les reporters, des jeunes, m'interrogeaient sur ce point avec le ton sceptique : "Comment Groulx, qui était un nationaliste traditionnel, peut-il avoir quoi que ce soit à

7 Texte paru dans L'Action nationale de mai 1978. 
faire avec le nationalisme d'aujourd'hui, si différent, si renouvelé qu'il n'a plus rien de commun avec le nationalisme conservateur d'autrefois? "Question fort intéressante d'ailleurs, mais qui ne fait qu'illustrer jusqu'où peut aller la confusion des idées entre le nationalisme, sa nature propre, son sens et sa portée, et le contenu culturel de ce nationalisme. Il est clair qu'on pensait - d'ailleurs on l'a précisé à un moment donné - à la forte insistance que mettait l'abbé Groulx sur le reflet "catholique " de son nationalisme et du nationalisme de son époque. Mais là n'est pas l'essentiel du nationalisme; et pas plus du nationalisme qu'il a lui-même proposé et enseigné, tout en estimant que dans notre cas à nous, dans notre situation propre, il y avait nécessité de rester fidèles, si nous voulions que notre avenir national soit assuré, aux allégeances catholiques dont notre culture a été si substantiellement tissée par les circonstances de notre histoire.

Autre chose est la définition des conditions dans lesquelles un peuple existe et a droit d'être reconnu comme existant pour les appliquer ensuite à une situation historique concrète et justifier des attitudes nationalistes (de défense d'une personnalité nationale); et autre chose, de définir le contenu culturel d'une identité nationale donnée pour tirer des conclusions sur l'opportunité historique d'en entreprendre la conversion vers autre chose. Même si Groulx a estimé que hors de nos fidélités catholiques, nos chances de salut étaient quasi nulles, et s'il a pu se tromper sur cela - la fin de toute cette histoire reste d'ailleurs encore à venir (!) - son oeuvre essentielle n'a pas du tout été là et n'est pas du tout dans la veine de confondre religion et nationalité. Bien au contraire, c'est une confusion dont il comprenait trop le sens et les conséquences pour qu'il y soit tombé. Certes, c'est en grande partie notre profonde imprégnation par le catholicisme traditionnel qui explique la différence qui nous justifie d'avoir des prétentions "nationales" face au protestantisme de l'anglo-canadianisme, et au pragmatisme de l'American Way of Life. Le risque que peut nous faire courir la volonté de modifier nos optiques ne nous enlève cependant aucun des droits nationaux que nous en avons tirés, ni ne crée aucun droit à «l'autre » de vouloir nous imposer sa façon protestante ou américaine d'évoluer vers de nouvelles formes de culture.

L'oeuvre essentielle de Groulx à ce sujet, c'est donc tout 
d'abord sa vision de l'histoire du peuple canadien-français et de son destin de peuple dans l'histoire. D'où au plan de l'action découle, pour ce peuple, un devoir humain et humaniste de s'accomplir pleinement par le souci de la "libération de la sujétion étrangère" et de "son essor souverain $"^{8}$. Pour illustrer tout cela, il parle de langue, de culture, de nation, de souveraineté, de libération, d'indépendance; et de religion aussi mais essentiellement dans la culture, à ce titre. Le nationalisme québécois d'aujourd'hui ne peut à son tour que parler de cela et de rien d'autre au plan fondamental. Sauf à démontrer que Groulx a mal formulé la question ainsi posée - et alors il aura fort à faire s'il est un vrai nationaliste - il reste tributaire, il le serait même s'il refusait de l'admettre, de tout ce qu'a formulé l'abbé Groulx sur l'ensemble de la question, sauf à prétendre que, contrairement à l'avis de Groulx, il ne voit aucun danger à émanciper notre culture traditionnelle de sa coloration catholique.

À partir du moment où le Québec d'aujourd'hui parle un langage national, exprime son destin en termes de nationalité et de nationalisme, et pour cela de libération, il ne peut que reprendre ce que Groulx a élaboré et qui n'a jamais cessé d'être de plus en plus présent dans notre langage politique au moins jusque vers 1950, même si d'aucuns de ceux qui parlent comme lui aujourd'hui, parlaient ou pensaient différemment dans les années 50. Autrement dit, la doctrine de Groulx va trop à l'essentiel, au fondamental, qui alors n'est pas uniquement de lui mais fait partie de l'héritage de la philosophie politique et sociale universelle, pour que l'effort d'application à notre cas qu'il en a fait, puisse être reformulé dans des termes qui n'auraient rien de commun avec sa pensée. N'importe quel examen objectif des textes de l'abbé Groulx, tant à L'Action française, que depuis dans de multiples conférences après cela, montrera que c'est l'arsenal de tous ses propos, à l'exclusion seule de la dimension proprement catholique, que nous utilisons et répétons aujourd'hui dans la lutte d'émancipation que nous menons; et aujourd'hui, non seulement dans le parti de l'indépendance, mais aussi bien dans tous les autres partis, dont le langage, si peu satisfaisant qu'il paraisse aux indépendantistes, a si considérablement changé par comparaison à ce que disaient les politiciens des

8 L'Action française (janvier 1921). 
«vieux partis » à partir de 1920 jusqu’à nos jours.

Or, ce qu'il est important de souligner ici pour notre propos, c'est que le dessein de Groulx était parfaitement lucide, comme nous l'avons vu par quelques références essentielles que l'on pourrait multiplier à loisir. Il a su ce qu'il voulait pour notre peuple et où il souhaitait que son action le conduise, sauf peut-être en ce qui concerne le régime politique précis dans lequel s'incarnerait l'accomplissement final de "l'essor souverain $»^{9}$. Quand il prend la direction de la revue $L^{\prime}$ Action française, en 1921, il fait le constat de notre situation en vue de déterminer ce qu'il faut faire. Il s'exprime alors en termes philosophiques, sur la perspective d'une nation tombée en état de déchéance et qu'il faut rééduquer. Et pour lui, en telle situation, se battre pour la survivance d'une minorité qui accepte son sort de minorité équivaut à accepter la mort, l'assimilation éventuelle. Il a décidé d'oeuvrer à l'intérieur d'une « ligue » qui a nom "Ligue des droits du français " et défend les droits de la langue, menacée à Montréal même. Il en fait changer le nom en Ligue d'Action française pour marquer qu'il y avait en jeu bien plus que la langue; une nation française.

Notre situation est devenue telle, écrit-il, dans ce texte fondamental dont nous avons tiré les quelques références précédentes, que «nous avons à franchir l'étape de l'individualité à la personnalité ». Qu'est-ce que cela signifie? Il en est en somme des peuples comme des individus, a-t-il expliqué :

Celui-là seul a gravi vers les vraies formes d'humanité qui a pu établir, par tout son être, la règle souveraine de la raison et de la liberté. Il est de même des peuples sans consistance morale, victimes d'une jeunesse ou d'une faiblesse trop originelle. Leur conscience, leur raison publique mal éveillées ne fournissent aucun pôle vers lequel s'orientent les énergies. Ces peuples se dirigent beaucoup moins qu'ils ne sont dirigés. Ils tombent dans la dépendance de tous les courants d'opinion; ils sont les vassaux des maîtres ou des voisins plus forts qui, de l'extérieur,

9 Voir à ce sujet la discussion des attitudes et du message de Lionel Groulx dans l'article de L'Action nationale de mai 1978. 
leur imposent leur domination morale ou politique. Ces peuples gisent encore ou sont retombés à la période de l'individualité. L'effort que leur destinée leur commande, c'est de se dégager de la sujétion étrangère et de l'inconsistance de leurs propres pensées; c'est de s'élever jusqu'à la personnalité nationale, jusqu'à l'état d'âme supérieur où ils prendront en euxmêmes, dans la synthèse de leurs vertus naturelles, dans le commandement de leur histoire et de leur vocation, le gouvernement immédiat de leur pensée, l'essor souverain de leur vie. C'est bien cette loi de nos destinées, principe et condition de la personnalité ethnique, doctrine où se fixe l'esprit collectif, que L'Action française s'est efforcée de mettre en lumière depuis sa naissance. ${ }^{10}$

Il se donne donc clairement, à lui et au mouvement d'action française, qu'il va diriger, une mission. Et la mission qu'il se donne, à partir de son rôle d'historien destiné à éclairer les voies de l'avenir à la lumière du passé, est non moins claire. Ce n'est pas celle d'un libérateur, mais d'un éducateur. Il s'agit d'abord de former avant de libérer, de former pour libérer, d'élaborer un programme pour «fortifier notre élément,... lui faire une riche et vigoureuse personnalité (de nation)", pour que "quoiqu'il advienne demain... notre peuple soit assez robuste... pour faire face à ses destinées ". Dès ce moment, il manifeste qu'il lui importe peu, dans l'immédiat du moins, que ce soit une Confédération qui «se reconstruit de nouvelles bases" ou "(qui) s'écroule», que ce soit dans "l'absorption impériale ou l'annexion américaine», ou dans "un État français (surgissant) du morcellement du continent ". Nous sommes un "petit peuple " et nous ne pouvons pas être assurés de gouverner notre destin exactement à notre guise en termes de solutions concrètes. Mais l'idée de Lionel Groulx, c'est que personne ne peut vaincre une personnalité nationale bien établie. Un sentiment national fort saura toujours profiter des circonstances propres à son accomplissement à travers les diverses difficultés et situations, et manouvrer pour atteindre ses fins ultimes en temps opportun dans l'histoire. De cette position et de cette orientation de base, il n'a en somme jamais dévié, quoiqu'en prétendent ceux qui disent pouvoir trouver dans l'oeuvre de Groulx tout ce qu'on peut vouloir, se contentant de bout de textes dont la partie circonstancielle est

\footnotetext{
702.

10 L'Action française (février 1921), cité dans L'Action nationale (mars-avril 1963):
} 
toujours de corriger les déviations que d'aucuns voudraient lui imposer par rapport à cette ligne fondamentale qu'il s'est tracée. C'est là son rôle de prêtre et d'historien dans la lutte nationale; aux autres de définir le leur.

Des expressions d'opinions de ce genre nous apparaissent aujourd'hui tellement normales, même en regard d'opinions divergentes qui leur reconnaissent quand même leur droit de cité, qu'on a peine à s'imaginer le pourquoi du degré d'hostilité auquel l'abbé Groulx fut en butte à l'époque. Et nombreux sont les jeunes des générations plus récentes qui n'arrivent pas à comprendre comment Henri Bourassa, dont on a tant vanté les mérites de champion de nos droits et exalté les qualités d'intelligence et d'indépendance d'esprit, ait pu s'insurger devant une telle attitude. On en vient même à faire l'injure à Bourassa de le classer parmi les fédéralistes inconditionnels de l'heure actuelle.

Bourassa, naturellement, c'était tout autre chose que cela. Son hostilité contre les propos de l'abbé Groulx était surtout tactique, comme il a été expliqué précédemment. Mais l'hostilité des Alexandre Taschereau, des Ernest Lapointe, des Duplessis première manière, et de toute la cohorte partisane qu'ils dirigeaient était, elle, foncière, viscérale, tellement tout cela les mettait en mauvaise posture dans leurs partis par rapport aux «Hautes Autorités " qui ne les toléreraient que si leur travail paraissait contribuer à notre «intégration " à plus ou moins long terme.

On haïssait l'abbé Groulx, et d'ailleurs aussi Bourassa, donc à fortiori et encore plus fort l'abbé Groulx, pour des idées dites "corruptrices de la jeunesse", "semeuses de haine des AngloCanadiens", etc., etc., en cela même que, pour ces gens, elles contribuaient à "réveiller le chat qui paraissait à la veille de s'endormir ". Or cette hostilité foncière du temps, quand on pense que ces idées se sont maintenant fait au moins leur place pour être acceptées comme étant aujourd'hui admissibles pour fin de discussion, donne une idée frappante de la portée de son influence par le grand chemin parcouru. 
Pourtant, la pénétration des idées de Groulx a été graduelle, très lente au début, puis en s'accélérant, toujours constante et manifeste. Vingt ans après le texte de 1921, en 1940, lui-même ne constatait guère de progrès. Il disait, dans une conférence à RoliynNoranda, en 1940:

Les Canadiens français ne sont pas encore résignés à mourir.

Sont-ils décidés à vivre? Je n'en suis pas sûr. Ils ressemblent à ces arbres qui ne sont pas encore morts, mais qui ont des branches sèches dans la tête et dont l'on ne sait pas s'ils vont reverdir le printemps prochain. Car enfin... si les Canadiens français ont décidé de vivre, vous paraît-il qu'ils en prennent les moyens? Vous paraît-il, oserai-je même dire, que cette affaire les préoccupe gravement?

Et à travers une sorte de parabole, il pose la question : "Les Canadiens français sont-il décidés à s'atteler pour le grand labeur...?" Il conclut: "Je voudrais bien qu'on pût me répondre: oui!"

C'est à travers les Sociétés Saint-Jean-Baptiste, dont il était l'inspirateur direct, et tout particulièrement de la Société SaintJean-Baptiste de Montréal, au Conseil d'administration où il a siégé, que la pensée de Groulx va se diffuser. Ces Sociétés ont servi comme d'antichambre à l'introduction des idées nationalistes dans les partis traditionnels. Une thèse faite à l'Université de Montréal a démontré que les idées développées par la Société Saint-JeanBaptiste de Montréal, auxquelles s'opposaient d'abord vigoureusement les deux partis traditionnels, se retrouvaient intégrées dans les programmes de ces partis environ dix ans après.

Et les Sociétés Saint-Jean-Baptiste elles-mêmes n'ont tiré que graduellement parti de tout l'arsenal des idées de Groulx. Composées d'éléments populaires émanant de la petite bourgeoisie canadienne-française, petite bourgeoisie qui a aussi ses intérêts et ses accointances avec la politique quotidienne, on y a toujours considéré Groulx comme une espèce de dieu; mais comme toujours avec les dieux, un dieu incarnant un idéal pur qui n'est pas fait en entier pour le commun des mortels. On y aménageait Groulx aux besoins du jour, mais en se réinspirant de lui à chaque étape pour faire face aux nouveaux besoins d'idées, d'arguments et de concepts qui ont conduit finalement aux prises de positions plus catégoriques, vers 
1965: le français seule langue officielle au Québec et la transformation de la Confédération en États associés, selon le principe de la souveraineté politique absolue du Québec.

Mais le rayonnement et l'influence de l'abbé Groulx, ce n'est pas seulement une pensée percutante, actuelle en ce sens qu'elle rejoint des virtualités profondes et qu'elle incite à l'actualisation, se propageant dans le milieu à partir d'une tour d'ivoire. C'est aussi une personnalité qui agit, intervient, provoque, attache et stimule à l'action. Tout d'abord, une forme d'éloquence qui n'est pas du tout celle du tribun ou du grand orateur, mais qui est imprégnée d'une conviction, d'un sens du tragique et d'une vibration émotive qui empoigne l'auditeur. La multitude des conférences qu'il a données, dans les circonstances les plus diverses et presque à tout venant - il suffisait qu'il soit requis pour que son sens de la mission à accomplir le commande - auront beaucoup fait pour rendre sa pensée vivante dans une multitude de têtes de disciples, de fidèles ou d'admirateurs. Toutes ou à peu près toutes sont axées sur l'histoire, sur les leçons de l'histoire, mais toujours mises en rapport étroit avec l'actualité même des circonstances dans lesquelles il parle.

C'était aussi une personnalité accueillante. Sa porte était ouverte à peu près à quiconque voulait le voir, demander des conseils, connaître son opinion avant de prendre une décision, ou simplement échanger des points de vue sur les problèmes de l'heure et savoir comment il les situait dans la perspective de ses connaissances historiques et de son nationalisme. Ayant commencé sa carrière comme professeur de collège, il aimait les jeunes et il était très proche d'eux. Ceux qui le désiraient, pouvaient le voir facilement, être reçus dans la plus grande simplicité et se retrouver finalement profondément marqués par lui. Chez lui, tout cela allait de soi parce qu'il concevait sa vie avant tout comme un apostolat. Et son espoir à travers toutes ces rencontres qui affluaient vers lui parce qu'il était considéré comme le grand conseiller et le grand éveilleur de la nation, c'était de découvrir et de stimuler à l'action l'homme, le "chef ", qui prendrait la direction effective du mouve- 
ment libérateur et fonderait le parti sauveur, celui qui nous dégagerait de la partisannerie politique du "bleu» et du "rouge", du conservateur et du libéral, irrémédiablement liés aux forces assimilatrices. Dans cette activité de contacts et de conseils, avec les espoirs qui le motivaient encore plus à l'ouverture d'esprit envers les personnes, il est notoire qu'il a joué un rôle actif, quoique discret, dans les essais préparatoires à la formation du Parti québécois qu'ont été l'Action libérale nationale et le Bloc Populaire ${ }^{11}$.

"Au sujet du Bloc Populaire, l'historien Robert Rumilly, toujours en défense de Maurice Duplessis, a cru bon, dans le film sur le chanoine Groulx préparé par Explo-Mundo, d'attribuer à celui-ci "l'erreur stratégique " qu'aurait commise le Bloc Populaire, de vouloir absolument porter sa lutte contre Duplessis, qui aurait pu autrement être un allié du Bloc contre Mackenzie King, le véritable adversaire du moment face à la conscription. Si je me souviens bien de ses propos, ce serait la haine qu'aurait entretenue le chanoine Groulx contre Duplessis qui l'aurait amené à forcer en quelque sorte les gens du Bloc Populaire à s'engager au provincial autant qu'au fédéral. À moins que M. Rumilly n'apporte des preuves formelles de ses prétentions, je suis fondé à croire qu'il accrédite là, contre l'abbé Groulx, une fort malveillante légende. Ả cette époque, j’étais très près du Bloc Populaire. Je travaillais très étroitement, jour après jour, avec André Laurendeau, alors directeur de L'Action nationale. J'étais également en contacts très fréquents avec Maxime Raymond. Laurendeau me rapportait au jour le jour ce qui se passait au Bloc et nous en discutions en fonction des décisions qu'il était lui-même sollicité de prendre. Jamais il ne m'a parlé de pressions de l'abbé Groulx en ce sens; et je n’ai jamais entendu l'abbé Groulx, aux réunions de L'Action nationale, prendre une attitude aussi tranchée sur le sujet. Ceux qui forçaient la main de Maxime Raymond pour étendre son action, très fortement contre son gré, au plan provincial, c'étaient le Docteur Philippe Hamel, René Chaloult et d'autres anciens de L'Action libérale nationale. Comme on peut le voir dans les pages du journal reproduites dans Hommage à Lionel Groulx*, les deux parties en litige ont cherché la médiation de l'abbé Groulx. Il s'est trouvé mêlé ainsi à la dispute, mais non comme avocat impérieux en faveur de l'une ou l'autre thèse. La conclusion de l'une de ces notes de journal, alors qu'il se propose d'écrire une dernière fois au Dr Hamel, montre plutôt qu'il n'approuvait pas la rigidité des attitudes de ce dernier; et c'était ce sujet précis de la participation obligatoire du Bloc au plan provincial qui faisait l'objet du litige autour duquel se jouait l'adhésion au Bloc des prestigieux anciens de L'Action libérale nationale. Cela est plus conforme à ce que j’ai connu de ces événements au cours desquels comme toujours, l'abbé Groulx avait avant tout à coeur que s’aplanissent les conflits internes entre «de fort braves gens, mais qui se laissent d'abord guider par leurs vieilles rancunes et par le sentiment ", et que naisse et réussisse le troisième parti. La scission s'opéra entre les deux groupes avant que les négociations fussent entièrement résolues, le Docteur Hamel n'ayant pas jugé assez ardents les sentiments antitrust de l'électricité de Maxime Raymond. À celui-là, il apparaissait que c'était surtout "la sympathie de la famille Raymond pour les «trusts " qui expliquait le souci de Maxime Raymond de ne pas s'engager dans la lutte provinciale, où il devrait se prononcer à ce sujet. Après la rupture, Maxime Raymond se résigna finalement à aller quand même au plan provincial, mais non pas parce que l'abbé Groulx le lui imposait ; parce qu'il craignait que le Docteur Hamel ne continue de lui faire tort en l'accusant d'être un suppôt des "trusts" et de refuser pour cela de combattre Duplessis. C'est alors qu'André Laurendeau fut amené à accepter la direction du Bloc à Québec, au lieu et place de René Chaloult, qui était le chef provincial envisagé avant la rupture.

* Maurice Filion, dir, Hommage à Lionel Groulx (Leméac, 1978), 200. 
Mais il y eut une période de sa vie, ses dix années de direction à L'Action française, où il mit au service de la cause, directement, sa personnalité d'animateur. Dans son rôle de directeur de la revue, il ne reçoit pas seulement des articles au gré de personnes désireuses de s'exprimer. Il anime au sens le plus plein du mot des équipes de travail, qu'il fait fouiller, chercher, penser, sur notre situation et nos problèmes. Il faut mieux définir les concepts, établir la situation concrète du peuple canadien-français, les problèmes auxquels il fait face, les obstacles qu'il rencontre à les résoudre, les moyens d'en sortir. Il en résultera tout spécialement cette admirable série d'articles échelonnés sur une période de dix ans sous la rubrique générale "Enquêtes".

Au bout de ces dix ans, nous l'avons déjà dit, l'abbé Groulx prendra sa "retraite" de cette forme directe d'action, dans l'intention de se confiner dans sa tâche d'historien, intention déjouée par la pression de ses admirateurs qui l'induira à assaisonner son travail scientifique des conférences, des entrevues et des contacts dont nous avons parlé. De ces dix ans d'action restera une oeuvre de base - la doctrine politique élaborée à L'Action française - et des disciples, dont un, en particulier, Esdras Minville, sera directement le continuateur du travail d'action intellectuelle entrepris. En succession à L'Action française et pour en continuer le travail, Esdras Minville fondera L'Action nationale en 1933, revue qui existe toujours.

L'on peut tout simplement annexer au bilan de l'influence de l'abbé Groulx, tout le travail et les influences mêmes que cette revue a exercés depuis: L'Action nationale ne fera pas autre chose, pendant toutes ses années d'existence, que de reprendre les bases doctrinales élaborées par l'abbé Groulx à L'Action française, afin de les repenser sans cesse, sans en dévier, en fonction de l'actualité des années qui suivirent. Sans doute, L'Action nationale y ajoutera des aspects nouveaux - comme une accentuation des aspects économiques par exemple, qui sont plus la spécialité de Minville -, mais toujours tissés sur le même fond inaltérable aux yeux de Minville comme de toute l'équipe.

Donc, indubitablement, un homme, Lionel Groulx, a repris en main par la pensée son peuple en pleine décadence, selon la perspective dans laquelle il voit son honneur et son destin. Sans lui, sa 
pensée et la projection qu'il a su en faire, nous ne pouvons vraiment pas présumer de ce que nous serions aujourd'hui.

Sur un point cependant, nous ne sommes plus ce qu'il aurait voulu que nous restions, et estimait que nous devions être et rester: un peuple fidèle, intimement fidèle aux dimensions catholiques qui avaient joué un rôle si fondamental dans la définition de son identité culturelle même. Pour lui, notre survie, nous l'avons déjà noté, se révélerait impossible si nous allions nous écarter de cette voie, puisque c'est notre identité même, dans la différence qui nous spécifie comme nation, qui disparaîtrait.

Au-delà de la décatholicisation - ce qui cependant pour lui n'empêchait pas la décléricalisation - du Québec, il ne voyait que l'assimilation. Nous croyons important de nous arrêter plus spécialement sur ce point en terminant, dans la mesure même où il sert à tort à reléguer l'influence de Groulx sur notre milieu à un rôle historique qui a pris fin avec l'avènement d'un supposé «nouveau nationalisme".

Effectivement - et peut-être en raison de la confusion qu'ils faisaient entre les deux - ceux qui combattaient notre nationalisme ont été aussi ceux qui combattaient notre catholicisme, lui préférant des éléments culturels plus imprégnés de neutralisme et même d'athéisme ou d'agnosticisme, et surtout de ce socialisme que l'Église condamnait.

Pendant la décennie 50, ils étaient des «collaborateurs " étroits du fédéral contre Duplessis et prêchaient l'«intégration lucide»du Canada français à un Canada où se mêleraient les deux cultures pour former une culture canadienne unique à doubles reflets. Leur comportement donnait entièrement raison aux craintes de l'abbé Groulx. Puis l'instinct reprit le dessus et le revirement fut brutal, laissant quelques-uns seulement des cités-libristes et alliés, - Jean Marchand, Gérard Pelletier, Pierre-Elliott Trudeau, Jean Pellerin - continuer seuls la route fédéraliste pour un Canada global. Or, fait étrange, la conversion se fait spontanément vers un nationalisme résolument indépendantiste, comme si à ceux-là venait la cons- 
cience, à bon escient, que la protection d'un État absolument libre d'user de sa souveraineté à sa guise était devenue indispensable à un peuple engagé dans une révolution culturelle. Il faut noter aujourd'hui que nous n'aurions pas de toute façon évité la crise d'identité dont Groulx voulait nous protéger en raison de ses convictions religieuses profondes d'abord, sans doute, mais au plan historique en raison des dangers qu'elle présente pour notre survie. Avec les transformations mêmes que s'est mis à connaître le catholicisme mondial et la grande marge d'incertitude qui en résulte sur ce que signifie le catholicisme comme religion même, et à fortiori comme définition d'identité culturelle, nous devions connaître les ébranlements qui nous ont atteints d'abord autrement.

Les deux thèses nationalistes qui s'affrontent alors sont du type suivant, l'une plus explicite, l'autre plus implicite. Celle de Groulx qui suppose au moins implicitement, et sans doute un peu plus, que nous ne pouvons pas sortir facilement de la Confédération, sauf si celle-ci se défait d'elle-même ou lorsque celle-ci se défera par le cours ordinaire de l'histoire. Notre force, et nos chances de salut, c'est alors notre identité culturelle propre dans laquelle il faut fortifier notre personnalité nationale grâce à quoi rien ne saurait prévaloir contre nous. Bref, un nationalisme avant tout culturel, faute de pouvoir être plus proprement politique; et parce que le fondement de tout nationalisme est d'abord culturel. Et toute cette thèse est chez Groulx fort explicite, appelant un haut degré de fidélité à la culture originelle. À quoi s'oppose celle des novateurs en matière de culture, qui se dégage plus de leurs évolutions que de leurs propos explicites sur l'indépendance du Québec. Ici un nationalisme d'abord politique, qui est le risque à prendre pour sauver la nation à laquelle on se révèle attaché (tout en se demandant pourquoi, comme le fit un jour Fernand Dumont ${ }^{12}$ ), mais dont on refuse pourtant d'accepter l'identité culturelle telle que l'a forgée l'histoire. On perçoit alors tout à coup qu'une telle mutation ne peut se faire sans grave danger d'assimilation sous le regard d'un occupant «qui manouvre les manettes politiques ".

En passant ainsi à un indépendantisme sur lequel Lionel Groulx s'est toujours abstenu de se prononcer carrément en termes

12 Entre autres articles au Devoir, cf Le Québec dans le Canada de demain (Ed. du Jour, 1967), $1: 188$ et ss. 
d'opportunité actuelle, les nouveaux nationalistes acquièrent d'autant plus l'impression de ne rien devoir à Groulx dont ils récusent la thèse de l'identité culturelle catholique qu'il nous objurgue de sauvegarder. Mais leur schéma de pensée nationaliste ne peut finalement pas s'articuler autrement qu'en fonction de la doctrine proprement nationaliste de Groulx, comme nous l'avons vu dans la première partie de ce texte. Et il reste que dans sa légitimité propre, au regard de la philosophie et de l'histoire, et par suite du regard sur nous des institutions politiques internationales, ce nationalisme politique ne peut reposer tout entier que sur la vision de l'histoire mise au point par l'abbé Groulx et l'identité culturelle qui a découlé de cette histoire, donc sur ce catholicisme même qui, avec la langue bien sûr et d'abord, nous avaient fait différents.

Dans ces deux thèses, il n'y a donc pas vraiment deux nationalismes différents en tant que nationalismes. Et le chanoine Groulx ne pensait pas autrement que cela, ayant éprouvé à l'égard du "nouveau nationalisme " un sentiment de grande satisfaction, tout en déplorant qu'il soit acatholique. Et d'autant plus que sur le plan politique, il ne trouvait pas convenable que l'on puisse envisager de sauver une nation de "la sujétion étrangère " en niant la validité de son identité culturelle, de sa "personnalité " nationale, et en entreprenant de la refaire sous les yeux de l'ennemi. Pour lui, «le premier devoir envers sa race, c'est d'en être ", avait-il déjà écrit ${ }^{13}$.

Quant aux sentiments propres de Lionel Groulx sur le sujet, la question est peut-être délicate à traiter en ce sens qu'il n'aurait peutêtre pas eu le goût d'intellectualiser sur ce propos et d'accepter ouvertement, ne fut-ce que pour des raisons de stratégie, qu'on pourrait, tout aussi bien être nationaliste au Canada français sans accepter l'identité culturelle catholique. Le fait qu'il n'avait pas du tout le goût de partir en guerre contre le "nouveau nationalisme" reste cependant un indice assez significatif de sa pensée profonde. Mais de toute façon, sa réponse, en termes de possibilité concrète de survie en telle occasion, eut été un non formel. Telle était sa croyance. Et il acceptait le nouveau nationalisme en espérant sa conversion éventuelle. Mais il n'aurait pas, il n'a jamais pour cela identifié, bien mieux confondu, nous l'avons déjà dit, nationalisme

13 Cité par Esdras Minville, dans Instruction ou Éducation (ESP, nos 204-205, Montréal, 1931) 49. 
et catholicisme comme étant liés de nécessité de nature.

Pour illustrer ce que nous avons développé à ce sujet au début du présent texte, prenons comme modèle de sa pensée le texte du message qu'il donnait à l'ouverture des États généraux du Canada français. Il le livre à un moment $(1966)^{14}$ où dans d'autres oeuvres de la fin de sa vie, il insiste hautement, et fortement, sur les dangers qu'il voit à la sorte d'apostasie à laquelle il assiste au sein de son peuple. Pourtant, l'aspect religieux ne vient qu'au dernier paragraphe du texte, introduit par un "enfin " qui le situe bien à sa place dans l'optique profane d'un geste politique, dans un ordre qui est celui de la qualité, non de la nature, comme un caractère spécifique de notre nationalisme.

Ce qui, sous ce chef, vient avant dans l'ordre, c'est :

1- la conscience de la nation résultant du même passé, des mêmes traditions, du même pays ;

2-un État doté du degré de souveraineté suffisant pour correspondre aux exigences de notre besoin d'être nous-même;

3-la liberté économique, base de la vie collective; avec ce que cela comporte pour nous de libération et de récupération;

4-un ordre social de prestige et de charité, qu'il identifie cependant ici plus spécialement pour nous, à un «ordre chrétien ";

5- le souci d'une culture à sauvegarder et qu'il définit comme française, sans ajouter "et catholique" dans cette énumération qui distingue les plans.

Après quoi vient en sixième lieu, le respect du sentiment religieux selon «la foi de nos pères".

Le catholicisme de Groulx, dans la formule nationaliste du "français et catholique" comme caractérisation de notre identité nationale telle qu'issue de notre histoire, ce n'est pas au plan de la pensée politique qu'il est embêtant. C'est au plan prophétique. Il " voit » que si nous sommes infidèles à cette caractéristique de notre avènement à l'état de nation, nous ne survivrons pas, vu le cadre et les circonstances dans lesquels nous sommes forcés de nous réaliser. Cela sera ou ne sera pas. C'est de l'ordre des visions de l'avenir 141.

14 Pour le texte, voir G.-E. Giguère, Lionel Groulx, biographie (Bellarmin, 1978), 
contre lesquelles nous sommes en grande partie impuissants si elles résultent d'une illumination vraiment prophétique ou/et d'une analyse correcte des différents coups qui, comme à une partie d'échecs, peuvent être joués sur la ligne du destin. Bien sûr, ceux qui pensent autrement, qui aiment autrement, continueront de considérer qu'il n'y a pas lieu de trop croire à ces dangers; et prendront allégrement - en fait ont déjà pris et avec pas mal de succès - le risque de tout changer à cet égard. Mais seule l'histoire révélera si Groulx était vraiment prophète ou si ce n'était qu'un homme de grande foi dont le jugement sur notre évolution historique restait purement subjectif.

Il y a dans son testament, un passage qui peut nous laisser croire que le tout "peuple français et catholique", était si profondément intégré pour lui qu'il ne se serait pas intéressé à son sort s'il eut été autrement ${ }^{15}$. Le fait en lui-même est concevable puisqu'en telle éventualité, le prêtre n'aurait pas eu la même perception d'une mission sacerdotale à accomplir dans une consécration à l'histoire et à l'éducation nationaliste d'un peuple; mais le fait lui-même ne doit pas être confondu avec ses convictions de citoyen membre de la communauté canadienne-française. Jamais dans ses réactions spontanées, Groulx n'a, à ma connaissance, exprimé, comme tant d'autres par rapport au socialisme et au marxisme, qu'il ne voudrait plus d'un État français au Québec si nous cessions d'être catholiques. À ce point de vue, quoique prêtre, - et peut-être parce que prêtre et bon théologien, donc connaissant mieux ces choses en profondeur - sa démarche était beaucoup plus assurée que celle de Henri Bourassa, dont on sait les scrupules et la tendance à trancher en faveur de la religion contre le nationalisme si les circonstances paraissaient commander un choix cornélien. Groulx regardait plutôt spontanément vers la synthèse à trouver au-delà de deux antithèses, qui ne pouvaient pour lui n'être que des apparences de contradiction.

C'est ce qui en faisait un esprit très audacieux, parmi les plus audacieux de son entourage dans les décisions ou les positions à prendre en matière de nationalisme. Quant aux hommes par exemple, ils ne demandaient pas de billets de confession, avant de montrer de la sympathie et d'appuyer les actions qui lui paraissaient 
susceptibles de réaliser son grand espoir d'un chef national libérateur ou assumant des fonctions dans des postes stratégiques pour l'avancement politique du peuple canadien-français. Dans certaines bornes bien sûr, il savait prendre des risques raisonnables et laisser à la Providence, à laquelle il croyait, le soin de voir à ses propres affaires. On a dit - et cela a causé de la surprise chez certains et je puis sûrement confirmer que c'est exact - qu'il éprouvait beaucoup de sympathie à l'égard de René Lévesque, avant même la fondation du Parti Québécois d'ailleurs, comme l'homme qu'il voyait monter pour être celui qui assumerait le rôle dont il désirait l'avènement. Pourtant, à cette époque surtout, il est notoire que René Lévesque n'était pas un homme de tout repos pour quiconque aurait espéré voir s'établir une sorte de nationalisme théocratique au Québec. Cela n'a pu être possible justement qu'en fonction de la séparation qu'il savait faire entre les exigences de la libération nationale, qui était pour lui un impératif, et la fidélité à un contenu culturel qui n'est pas, en définitive, une affaire de "chef" mais de convictions populaires profondes.

De même, on a vu dans ses écrits avec quelle force il condamne l'attitude des évêques canadiens d'avoir sacrifié l'A.C.J.C., association nationaliste de jeunesse, pour bâtir des mouvements d'action catholique d'où toute perspective nationale sera exclue par définition. Loin d'estimer, comme Bourassa, encore une fois, que la religion pouvait nous demander le sacrifice de notre nationalisme, il croyait au contraire que la dénationalisation du peuple canadienfrançais aux fins de servir de supposés intérêts supérieurs de la religion, ouvrirait quasi fatalement la porte à la décatholicisation de ce peuple. Et les événements lui ont profondément donné raison au moins en apparence. Sa vision des choses, à ce sujet, c'est que toutes les Églises, et l'Église catholique plus que toute autre, doivent tout d'abord respecter les traditions et les cultures des peuples, tout en les catholicisant.

Ainsi apparaît-il que l'influence de Groulx sur son milieu persiste aujourd'hui autant qu'hier comme un fait actuel que nous vivons avec plus d'intensité que jamais; et non pas comme un fait 
historique à rappeler pour mémoire seulement. Il faut alors revenir, ici, pour qualifier l'influence de Groulx dans sa plénitude, au texte de l'article de L'Action nationale de mai 1978: Groulx, à la façon de Ghandi en Inde, aura été le libérateur de son peuple de la «sujétion étrangère ". Cette libération, il la souhaitait ardemment, il l'a préparée amoureusement; et le cours de notre histoire en a été infléchi de telle façon que le mouvement en ce sens semble maintenant irréversible, et se développe dans des conditions qui laissent présager le succès. 